\title{
ECG biometric authentication based on non-fiducial approach using kernel methods
}

\begin{abstract}
Identity recognition faces several challenges especially in extracting an individual's unique features from biometric modalities and pattern classifications. Electrocardiogram (ECG) waveforms, for instance, have unique identity properties for human recognition, and their signals are not periodic. At present, in order to generate a significant ECG feature set, nonfiducial methodologies based on an autocorrelation (AC) in conjunction with linear dimension reduction methods are used. This paper proposes a new non-fiducial framework for ECG biometric verification using kernel methods to reduce both high autocorrelation vectors' dimensionality and recognition system after denoising signals of 52 subjects with Discrete Wavelet Transform (DWT). The effects of different dimensionality reduction techniques for use in feature extraction were investigated to evaluate verification performance rates of a multi-class Support Vector Machine (SVM) with the One-Against-All (OAA) approach. The experimental results demonstrated higher test recognition rates of Gaussian OAA SVMs on random unknown ECG data sets with the use of the Kernel Principal Component Analysis (KPCA) as compared to the use of the Linear Discriminant Analysis (LDA) and Principal Component Analysis (PCA).
\end{abstract}

Keyword: ECG biometric recognition; Non-fiducial feature extraction; Kernel methods; Dimensionality reduction; Gaussian OAA SVM 